\section{Benign intracranial hypertension and Cushing's disease}

Benign intracranial hypertension is found typically in obese young women, often associated with parity, the contraceptive pill, and other changes in hormonal state. ${ }^{1}$ More rarely the syndrome is seen as a complication of Addison's disease, during withdrawal of corticosteroids, and in treatment with drugs such as nalidixic acid, nitrofurantoin, tetracycline, and indomethacin. An association with metabolic disorders such as carbon dioxide retention, hyponatraemic states and lead poisoning is also well known. A case is presented where benign intracranial hypertension was confirmed in a man with an adrenocorticotrophin-secreting pituitary microadenoma.

\section{Case report}

A constitutionally obese 18-year-old man was referred for investigation of delayed puberty. He had had occasional severe generalised headaches but no other complaints. He was of short stature (height $149.5 \mathrm{~cm}$ ), grossly obese (weight $110 \mathrm{~kg}$.), had scant body hair, prepubertal external genitalia, and a high-pitched voice. He had livid abdominal striae and predominantly centripetal fat distribution but no evidence of proximal myopathy or hypertension. He had bilateral papilloedema, confirmed by fluorescein angiography, but normal visual fields and acuity. Skull $x$-ray examination was normal and computerised tomographic (CT) scan and metrizamide cisternography, utilising CT scanning, showed normal ventricular size and no structural abnormality. A series of lumbar punctures confirmed benign intracranial hypertension, producing normal fluid at pressures between 20 and $40 \mathrm{~cm} \mathrm{H}_{2} \mathrm{O}$. Full blood count, urea and electrolytes, blood gases, and glucose tolerance tests were normal. Skeletal radiographs showed a retarded bone age of 15 years. Steroid measurements showed raised urinary free cortisol concentrations $(2900-4500 \mu \mathrm{mol} / 1 \quad(95: 12-163 \cdot 13 \mathrm{ng} / 100 \mathrm{ml})$, reference range $550(20)$ ), loss of diurnal variation of plasma cortisol, absent suppression of steroid secretion with low-dose dexamethasone, partial suppression during high-dose dexamethasone treatment, and an exaggerated 17-hydroxycorticosteroid response during a metyrapone test. Pituitary driven Cushing's disease was confirmed by inappropriately high concentrations of ACTH. Growth hormone (GH) deficiency was shown by undetectable GH concentrations during insulin-induced hypoglycaemia. He was hypogonadal with a low plasma testosterone concentration $(2.5 \mathrm{nmol} / 1$ $(7 \cdot 2 \mathrm{ng} / 100 \mathrm{ml})$ reference range $9-24(25 \cdot 9-59 \cdot 1))$, and diminished luteinising hormone ( $\mathrm{LH})$ and follicle-stimulating hormone (FSH) response to luteinising hormone-releasing hormone (LHRH). Prolactin concentrations, thyroid function, and urinary concentrating ability were normal.

After completing the endocrine investigations adrenal blockade was achieved with metyrapone and aminoglutethimide. Within four weeks of starting the blockade the patient's hypogonadism resolved, testosterone rising to $18.6 \mathrm{nmol} / 1(53.2 \mathrm{ng} / 100 \mathrm{ml})$. During adrenal blockade, however, he developed disabling raised-pressure headaches, which were not relieved by serial lumbar punctures and diuretic therapy, and the papilloedema progressed. Repeat CT scans were unchanged. A ventriculoatrial shunt was inserted and the headache and papilloedema resolved. After a period of strict dietary restriction while on adrenal blockade, during which he lost $25 \mathrm{~kg}$ in weight, a two-stage bilateral adrenalectomy was carried out. Nine months later, on replacement doses of corticosteroids, GH release in response to hypoglycaemia was normal, linear growth had resumed, genital growth had occurred, and his voice had broken. The pituitary-gonadal axis and thyroid function remained normal. Plasma ACTH concentrations had risen to expectedly high concentrations (453-526 $\mathrm{ng} / 1 \quad(20.56-23.88 \mathrm{fg} / 100 \mathrm{ml})$ reference range 20-80 (0.9-3.6)) without clinical evidence of Nelson's syndrome. We propose to give external pituitary irradiation when linear growth has ceased.

\section{Comment}

We know of no previous report of a relationship between benign intracranial hypertension and Cushing's disease. Probably in our patient longstanding and relatively mild intracranial hypertension, secondary to hypercortilism, was exacerbated by acute alteration of corticosteroid metabolism during adrenal blockade. The association of benign intracranial hypertension and other disordered states of adrenal function (Addison's disease, steroid withdrawal, obesity) ${ }^{2}$ is well recognised and it is thought that alteration of the CSF absorption gradient across the arachnoid villi may be the primary factor in resetting intracranial fluid compartments. ${ }^{3}$ We suggest that in our patient the high circulating levels of adrenal steroids and their abrupt reduction disturbed cerebral volume control and thus produced the manifestations of raised intracranial pressure.

${ }^{1}$ Donaldson JO. Neurology of pregnancy. In: Walton JN, ed. Major problems in neurology, vol 7. Philadelphia: WB Saunders, 1978:171-5.

2 Paterson R, De Pasquale N, Mann S. Pseudotumour cerebri. Medicine $1961 ; 40: 85-9$.

3 Johnston I, Paterson A. Benign intracranial hypertension. Brain 1974 ;97: 301-12.

(Accepted 28 March 1980)

Newcastle General Hospital, Westgate Road, Newcastle upon Tyne NE4 6BE

$P$ K NEWMAN, MB, MRCP, senior neurological registrar

M SNOW, MB, MRCP, consultant physician

P HUDGSON, MB, FRCP, consultant neurologist

\section{Platelet, granulocyte, and HLA antibodies in renal dialysis patients transfused with frozen blood}

Prior blood transfusion is believed to enhance renal graft survival in both live-related and cadaver graft recipients. Nevertheless, transfusion may induce the production of lymphocytotoxic antibodies, and there is controversy about the importance of these in graft survival. We have operated a policy of minimal blood transfusion in our regular dialysis programme and of using, when possible, frozen washed red cells (frozen blood). Our original aim in using frozen blood was to reduce the risk of transmitting hepatitis, but we also hoped that the lower leucocyte and platelet contamination of frozen blood would reduce HLA alloimmunisation. Owing to this policy we have been able to study a group of patients who have received frozen blood as the only antigenic stimulus. Previous studies ${ }^{12}$ have concentrated on the development of lymphocytotoxic (HLA) antibodies in such patients, whereas our study also examines the sera of these patients for platelet and granulocyte specific alloantibodies.

\section{Patients, methods, and results}

We studied 34 patients maintained on regular dialysis treatment (RDT) for between 1 and 106 months (mean 34 months). Fifteen patients had had no antigenic stimulus such as a blood transfusion, pregnancy, or failed renal graft, and they served as a negative control group. The remaining 19 patients were transfused with frozen blood as the only antigenic stimulus. They received an average of 5 units each (range 1-12 units). Blood was frozen by a low-glycerol liquid nitrogen technique and processed for transfusion as described by Jenkins and Blagdon. ${ }^{3}$ All serum samples were screened for platelet and leucocyte antibody. Tests included the standard lymphocyte cytotoxicity test (LCT) and the lymphocyte (LIFT), granulocyte.(GIFT), and platelet (PIFT) immunofluorescence tests developed at the Central Laboratory of the Netherlands Red Cross Blood Transfusion Service in Amsterdam.

The control group of 15 patients without antigenic stimulus had negative results on antibody screening: they were excluded from subsequent calculations. Nine $(47 \%)$ of the 19 patients transfused with frozen blood had antibodies as determined by a positive result in one or more of the screening tests (LCT, LIFT, GIFT, PIFT). When considered in terms of the methods used the results were positive with the LCT or LIFT, or both (indicating HLA specificity) in only two (10\%) of these 19 patients. A further seven patients $(37 \%)$ were alloimmunised to platelet specific (PIFT positive) or to granulocyte specific (GIFT positive) antigens, or to both. In these patients the LCT and LIFT were both negative.

\section{Comment}

A recent study has shown that a red cell suspension prepared from frozen blood is effectively depleted of leucocyte and platelet contamination. ${ }^{4}$ Nevertheless, our study has shown that the residual material can immunise the recipient against leucocyte and platelet 